\title{
En defensa de la teoría del desarrollo
}

\author{
José Antonio Alonso \\ Catedrático de Economía Aplicada \\ Director del ICEI*
}

\section{Resumen}

El artículo pretende identificar aquellas contribuciones de la teoría del desarrollo que pueden ser de interés para el enriquecimiento de la teoría del crecimiento. Para ello revisa las aportaciones básicas de la teoría de crecimiento y discute el grado en que sus prescripciones se corresponden con la realidad. Analiza después las contribuciones básicas de la teoría del desarrollo, tratando de extraer sus contribuciones más originales. Termina apuntando algunas muestras de cómo la teoría del crecimiento integró algunos postulados procedentes de la teoría del desarrollo.

Palabras Clave: Teoría del crecimiento, teoría del desarrollo, Crecimiento endógeno, trampas de pobreza, externalidades, equilibrios múltiples.

Clasificación JEL: 010, 011, 040.

\section{Abstract}

The article aims to identify those contributions to the development theory that could be of interest to enrich the growth theory. Therefore it reviews the basic contributions to growth theories and debates to what extent its prescriptions fit with reality. It follows an analysis of the basic contributions to development theories, trying to take the most original ones out. The article ends with some samples on how the growth theory has incorporated some of the postulates of the development theory.

Keywords: Development theory, growth theory, endogenous growth, poverty traps, externalities, multiple equilibria.

Classification JEL: 010, 011, 040.

\section{Introducción}

El intento de explicar las fuentes del progreso material de las naciones está en el centro de las preocupaciones básicas de los fundadores del pensamiento económico. De hecho, buena parte del esfuerzo analítico de Smith, Ricardo o Marx, se dedicó a entender las leyes que rigen el comportamiento dinámico de las economías en el largo plazo. La revolución marginalista, en la segunda mitad del siglo XIX, produjo un desplazamiento notable en el centro de la investigación económica, que pasó a volcarse, dominantemente, en definir los criterios de decisión óptima de los agentes y en argumentar las condiciones del equilibrio en los mercados. Su preocupación se orientó a fundamentar una asignación eficiente, en el corto plazo, más que en explicar la dinámica agregada de la economía, en el largo plazo. Como consecuencia, el crecimiento económico desaparece de las preocupaciones más centrales de la agen-

\footnotetext{
${ }^{*}$ Instituto Complutense de Estudios Internacionales.
} 
da investigadora en economía, desde 1870 a 1950. Existen excepciones, entre las que cabe destacar la aportación de Young (1928), sobre el papel de los rendimientos crecientes, y la original contribución de Schumpeter (1942), subrayando la influencia que el cambio técnico tiene en la dinámica económica y la función central que el empresario desempeña en ese proceso.

No será, sin embargo, hasta la década de los cincuenta del pasado siglo cuando la explicación de la dinámica económica de lago plazo vuelva a ocupar un puesto relevante en la reflexión económica. Desde sus mismos orígenes, este esfuerzo se canalizó a través de dos grandes tradiciones analíticas claramente diferenciadas: la teoría del desarrollo y la teoría del crecimiento. La primera se propuso construir un cuerpo teórico específico para el análisis de las condiciones de progreso de los países en desarrollo a partir de la consideración de las características deficiencias estructurales de este tipo de países; la segunda intentó generar una teoría de validez universal acerca del crecimiento económico. Mientras la primera recurrió a una forma de hacer economía preferentemente argumentativa y verbalizada, la segunda optó por traducir en modelos matemáticos sus intuiciones básicas. Esta opción otorgó una ventaja indudable a la teoría de crecimiento, que acabó por convertirse en la interpretación central de la dinámica económica. Ambas tradiciones, sin embargo, vivieron de espaldas la una de la otra a lo largo de buena parte de las últimas cinco décadas. Los economistas del desarrollo consideraban las propuestas de la teoría del crecimiento como excesivamente simplificadoras, abstractas y alejadas de la realidad; mientras los economistas del crecimiento estimaban que los economistas del desarrollo ofrecían una retórica sociológica de baja consistencia analítica y limitada capacidad de validación empírica. Como consecuencia, hasta muy recientemente, la comunicación entre estas dos tradiciones fue más bien escasa, para perjuicio de ambas.

En la actualidad, sin embargo, las diferencias entre estas dos tradiciones ha tendido a diluirse: los economistas del desarrollo valoran hoy en mayor medida la importancia de integrar sus propuestas en modelos económicos consistentes, capaces de ser sometidos a prueba empírica, asimilando su lenguaje al propio de la teoría del crecimiento; y, a su vez, los teóricos del crecimiento han pasado a buscar explicaciones más complejas, contemplando algunas dimensiones sociales e institucionales, que estaban antes alejadas de sus preocupaciones. Conviene decir, sin embargo, que no siempre este último proceso se produjo con el debido reconocimiento de las iniciales autorías de las ideas asumidas. Pero, más allá de este hecho, se han generado áreas cada vez más dilatadas de coincidencia y de mutua relación entre ambas tradiciones; un espacio por el que transitan las aportaciones de autores como Stiglitz, Sen, o Rodrik, por citar tres bien conocidos.

El presente ensayo trata de dar cuenta de ese proceso, poniendo especial énfasis en las ventajas que la explicación del crecimiento derivaría de una más atenta consideración de alguna de las intuiciones generadas por la teoría del desarrollo. Semejante ejercicio ayudaría a mejorar la explicación de la dinámica económica, incorporando de forma más sólida la posibilidad de relaciones no lineales, de cambios en la composición del output y de la eventual existencia de múltiples equilibrios. Adi- 
cionalmente, esa misma recomendación serviría para evitar que, en ocasiones, se nos presenten como enteramente novedosas propuestas que tienen una larga tradición en la investigación del desarrollo.

Para conocer el grado de originalidad de las aportaciones de la teoría del desarrollo, conviene referirse, previamente, siquiera de forma sumaria, al núcleo argumental de la teoría del crecimiento. A ello se dedica el siguiente epígrafe.

\section{La teoría del crecimiento}

\subsection{Planteamientos teóricos}

La moderna teoría del crecimiento se erige sobre la base de definir las condiciones de un estado estacionario (steady state) en el que se haga compatible el crecimiento del PIB agregado (o del PIB per cápita) con la preservación de la condiciones del equilibrio de la economía en el largo plazo. El crecimiento, a su vez, se entiende como un proceso lineal de expansión continuada del PIB per cápita, expresado a través de un flujo de bienes cuya composición, en la mayor parte de los modelos, se considera irrelevante. De ese esfuerzo analítico se deriva tanto la determinación de los factores promotores del crecimiento como la explicación de la dinámica de acercamiento al estado estacionario: ambas planteadas como propuestas de alcance universal, cualesquiera que sean las condiciones de los países implicados. En el marco de este esfuerzo analítico, la teoría del crecimiento ha avanzado a través de generaciones sucesivas de modelos, cada una de ellas tratando de eludir o superar los problemas de la generación anterior. Visto en perspectiva, sería un ejemplo notable de progreso científico, al modo en que lo teoriza Laudan (1977).

Pues bien, para la primera generación de modelos, construida por Harrod (1939 y 1948) y Domar (1946), la variable que determina el crecimiento económico es la inversión: ésta aparece determinada por la propensión a ahorrar, que incide en la expansión del producto condicionada por la productividad media del capital (la inversa de la relación capital-producto). Si se supone que esta última variable es relativamente inalterable en el tiempo (aunque cambiante entre países), será la capacidad de ahorro y, por tanto, la inversión resultante la que determinará la posibilidad de crecimiento de la economía. En la interpretación de Harrod, la inversión tiene la doble función de ampliar la capacidad productiva de una economía y de impulsar la demanda requerida para ese crecimiento. Este planteamiento parecía conforme con la experiencia de muchos estudios empíricos, que asociaban la transición de las economías a un régimen de rápido crecimiento con el logro de un incremento sostenido en sus tasas de ahorro e inversión. No obstante, dado que se partía de una relación estable entre capital y producto, el mantenimiento del pleno empleo de los factores - una exigencia de la condición de equilibrio- obligaba a que las tasas de crecimiento del producto y del stock de capital coincidiesen con la propia de la población. Es decir, en equilibrio el PIB per cápita permanece estacionario, salvo que 
se acepte la existencia de un progreso técnico que amplificase, de modo continuado, la productividad del trabajo. Aunque no se explica la procedencia de este progreso técnico, se supone que aparece asociado a la creciente acumulación de capital que la dinámica de crecimiento promueve.

La versión simplificada de la dinámica económica que ofrecía el modelo HarrodDomar constituyó una guía muy manejable para los estrategas del desarrollo, al ofrecer un marco razonado para la programación del crecimiento y una identificación de las variables relevantes sobre las que asentar la intervención económica ${ }^{1}$. En realidad, la clave consistía en encontrar la financiación adecuada para nutrir los niveles de inversión que se requerían para alcanzar el crecimiento deseado. Así, por ejemplo, si un país tenía una ratio capital-producto de 5 y la economía deseaba crecer a una tasa del 6\%, necesitaría alcanzar una tasa de ahorro (y de inversión) equivalente al 30\% del PIB. Es este enfoque el que nutre las primeras mediciones acerca de las necesidades de financiación de los países en desarrollo, como las debidas a Milikan y Rostow (1957) o a Rosestein-Rodan (1961), entre otros; sobre este mismo enfoque se construye, en esencia, el modelo de dos brechas, debido a Chenery y Strout, (1966), que inspiró la determinación de la ayuda internacional requerida para asentar un crecimiento sostenible en los países en desarrollo; y este mismo planteamiento fundamenta el modelo RMSM, que crea en 1972 el Banco Mundial para definir las necesidades financieras requeridas por los países en desarrollo para alcanzar un ritmo de crecimiento dado.

Hay otras dos consecuencias del modelo Harrod-Domar que resultan más inquietantes. La primera es que la historia cuenta: cualquier perturbación o accidente que sufra la trayectoria de crecimiento de un país tiende a perpetuarse en el tiempo, ya que no existe dinámica de convergencia al estado estacionario. Dicho de otro modo, aún cuando dos economías, una pobre y otra rica, tengan similares parámetros básicos (propensión a ahorrar y relación capital-producto), la diferencia entre las dos economías se sostendrá indefinidamente en el tiempo. La segunda es que la condición de equilibrio del modelo resulta improbable e inestable. Improbable, porque todas las variables relevantes en la fijación del estado estacionario son exógenas y sin relación definida entre ellas; e inestable, porque una perturbación respecto a la senda de equilibrio puede dar origen a un proceso que se amplifique en el tiempo, sin posible corrección.

La segunda generación de modelos de crecimiento, planteados en la década de los cincuenta, tratará de dar solución a este último problema. Para ello, las nuevas propuestas convierten en variable endógena alguno de los parámetros relevantes en la determinación del crecimiento económico. Así, por ejemplo, Kaldor (1957) convierte en variable la propensión a ahorrar, que evoluciona de acuerdo con la distribución del ingreso agregado. Por su parte, en la que será la propuesta canónica de esta etapa, Solow (1956 y 1957) y Swan (1956) convierten en variable endógena a la relación capital-producto, al admitir la sustituibilidad de factores en la función agrega-

${ }^{1}$ Domar, no obstante, se distanció de esta utilización del modelo y, de hecho, respaldó el modelo de Solow como una más adecuada explicación del crecimiento. 
da de producción. De este modo, el modelo identifica una condición de equilibrio (el estado estacionario) a la que cabe acceder y que es, además, estable. Esa condición de equilibrio queda determinada por parámetros (variables exógenas, por tanto) como la propensión a ahorrar, la tasa de crecimiento de la población o la tasa de progreso técnico. Adicionalmente, el modelo contempla la existencia de una dinámica de convergencia hacia el estado estacionario: el crecimiento es tanto mayor cuanto más alejada esté la economía de su senda de equilibrio a largo plazo, atenuándose a medida que se acerca a ese objetivo. Este proceso sugiere la progresiva igualación de los niveles de renta per cápita de todas aquellas economías que compartan estado estacionario, cualquiera que sea su nivel de desarrollo de partida. Posteriormente, autores como Cass (1965) o Koopmans (1965) desarrollaron este modelo suponiendo que los agentes tienen una conducta intertemporal óptima, otorgando con ello un desarrollo analítico más completo al enfoque.

Pese a su elegancia formal, el modelo de Solow-Swan arrastra algunas consecuencias paradójicas. La primera es que la tasa de ahorro influye en la determinación del nivel de renta de equilibrio, pero no tiene papel alguno en la promoción del crecimiento de la economía, una vez alcanzado el estado estacionario. Un resultado, este último, que resulta abiertamente contrario a lo que parece dictar la intuición económica; y que contrasta con lo prescrito por el modelo Harrod-Domar. El segundo resultado paradójico deriva del supuesto de rendimientos marginales decrecientes de los factores sobre el que se asienta la función de producción agregada. En ausencia de progreso técnico, el crecimiento de una economía depende de la capacidad para incrementar su nivel de capitalización. No obstante, a medida que se incrementa la ratio capital por persona, las posibilidades de crecimiento del PIB per cápita se atenúan en virtud de los rendimientos marginales decrecientes del capital, hasta llegar a su total extinción en el estado estacionario. El problema se acentúa por el hecho de separar, como si se tratase de dos dinámicas claramente diferenciadas, la capitalización de la economía y el progreso técnico, un hecho cuestionado, entre otros, por Kaldor (1957), Kaldor y Mirrless (1962) o, más tarde por Scott (1989).

Así pues, en las dos primeras propuestas (Harrod-Domar y Solow-Swan), el dinamismo del PIB per cápita del estado estacionario queda determinado, en última instancia, por la misma variable: la tasa de progreso técnico. No obstante, esta variable no se integra en ninguno de estos modelos, considerándose un factor ajeno a la explicación económica. Este tratamiento que se otorga al progreso tecnológico es, de hecho, el principal inconveniente del modelo Solow-Swan. Un inconveniente que se vio amplificado cuando las estimaciones empíricas del modelo ponían en evidencia el peso que el progreso técnico (incremento de la productividad total de los factores) tenía en la explicación del crecimiento económico de Estados Unidos (cuatro quintas partes del total, en el período 1909-49) (Solow, 1957). Resultaba abiertamente paradójico que la variable que se comprobaba como más relevante en la dinámica de progreso quedase desalojada de la explicación teórica del crecimiento.

Para aminorar la dimensión de este resultado paradójico se intentó enriquecer la contabilidad del crecimiento, bien sea cualificando los factores de producción o bien 
añadiendo factores adicionales en la explicación del crecimiento. De todos estos ensayos, el que más incidencia tuvo en el desarrollo posterior de la doctrina fue el debido a Makiw, et al. (1992), que amplían la función de producción agregada para integrar el capital humano como factor productivo adicional. La presencia de este factor permite encontrar, en un análisis cross-country, con una muestra 98 países, una explicación del crecimiento cercana al $80 \%$ de la variación en la renta de los países. No obstante, también en este caso el progreso técnico (y el propio esfuerzo en logros educativos) permanece como exógeno.

Para superar esta limitación nacieron las distintas familias de modelos de crecimiento endógeno, desplegados tras la primera mitad de la década de los ochenta. A todos ellos es común la búsqueda de un factor que reúna al tiempo dos condiciones: en primer lugar, que siendo un producto del crecimiento sea, a la vez, causa del crecimiento; en segundo lugar, que no esté sometido a rendimientos marginales decrecientes. En la mayor parte de los casos, se identifica el conocimiento con ese factor que se está buscando. Lo que diferencia, en todo caso, a los diversos modelos es el modo en que se corporeiza el conocimiento y la forma que adopta la función de producción en el que ese factor se integra. A este respecto, y sin agotar la relación, cabría distinguir cinco principales formas de tratar el conocimiento en el seno de los modelos de crecimiento endógeno. Por ejemplo, Rebelo (1991) presupone una función de producción lineal compuesta por un único factor, el capital, que agrega capital físico y humano; Romer (1986), siguiendo los desarrollos de Arrow (1962), considera que el progreso técnico se genera como consecuencia de las externalidades asociadas al proceso de acumulación de la inversión, a través de una dinámica de aprendizaje (learning by doing) y de efectos de derrame (knowledge spillover); Lucas (1988), siguiendo a Uzawa (1965), divide la economía en dos sectores, uno productor de bienes finales y otro de capital humano, haciendo que la complementariedad entre capital físico y capital humano libere de los rendimientos marginales decrecientes al esfuerzo inversor; Romer (1990) o Grossman y Helpman (1991) consideran que el dinamismo económico descansa en la existencia de un sector productor de ideas (de innovación, en suma) que opera en condiciones de competencia imperfecta y brinda una gama creciente de input al sector productor de bienes finales; y, en fin, Aghion y Howitt (1992 y 1998) o Grossman y Helpman (1991) consideran que el sector productor de ideas promueve un proceso de upgrading en la composición técnica de los bienes generados, desplazando los bienes previos (destrucción creativa) y mejorando la capacidad productiva del conjunto de la economía. No en todos estos modelos está garantizada una dinámica de convergencia hacia un estado estacionario; en algunos se presenta un fenómeno de escala en la determinación del crecimiento de la renta de equilibrio; y hay algunos en los que la presencia de un planificador (acción regulatoria del Estado) podría, al menos en teoría, mejorar las condiciones de eficiencia agregada.

Aun cuando no siempre el modo de tratar el progreso técnico en estos modelos resulta convincente, no cabe duda que se trata de propuestas que intentan captar de un modo más apropiado lo que constituye la experiencia del crecimiento económi- 
co. Sigue, no obstante, vigente el dilema que en su momento señaló Solow (1994) respecto a los modelos de crecimiento endógeno: si hay rendimientos crecientes de capital sin restricciones, la forma de la función de producción garantiza un output infinito en un período de tiempo finito; y si los rendimientos de escala son crecientes, pero los rendimientos de capital decrecientes, las implicaciones de los modelos no son muy distintas del modelo originario que él mismo formuló. Lo que deja una senda muy estrecha -expresada en valores muy exigente para los parámetros- a aquellas propuestas que aceptan la linealidad en los rendimientos de capital.

Si uno considera en perspectiva la evolución de la teoría del crecimiento advertirá tres rasgos básicos en su proceso de cambio:

i) en primer lugar, se ha avanzado hacia una mayor complejidad en la explicación de la dinámica económica, incorporando nuevos factores y nuevas condiciones económicas (presencia de competencia imperfecta, por ejemplo);

ii) en segundo lugar, en la identificación de los factores promotores del crecimiento se ha pasado del ámbito de lo tangible (capital físico) a otro en donde tienen mayor presencia factores menos tangibles, como las externalidades, el conocimiento, la tecnología o el capital humano (en alguna medida, se ha pasado de atender en exclusiva al hardware a considerar, también, el software de una economía); y

iii) la evolución de este cuerpo de doctrina se presenta como una sucesión de modelos que acogen factores diversos de explicación del crecimiento, sin que haya una clara sugerencia acerca de su carácter complementario (o alternativo).

Ahora bien, ¿qué grado de respaldo empírico tiene este esfuerzo teórico?

\subsection{Problemas empíricos}

No es el propósito de este epígrafe hacer un recuento de los ejercicios de validación empírica de la teoría del crecimiento: existe una abundante literatura al respecto (véase, por ejemplo, Durlauf y Quah, 1999 o Temple, 1999, entre otros). De lo que se trata, más bien, es de extraer una impresión genérica acerca del grado de acomodación entre el mundo al que remiten las diversas explicaciones teóricas del crecimiento y la realidad económica. Si la teoría es conforme con la realidad, debiera dar, cuando menos, una explicación de los notables y persistentes niveles de desigualdad que rigen en los niveles de desarrollo entre países y de la dinámica de crecimiento que se observa en la economía internacional. Desde esta perspectiva, no cabe duda que la valoración de la teoría disponible no puede ser excesivamente entusiasta.

Pese a su atractivo formal, el modelo neoclásico más simple encuentra problemas serios para superar la prueba. En concreto, si se admite que la tecnología constituye un bien público al que acceden los países sin coste, como supone de forma 
voluntariamente simplificada Solow (1956), todos los países debieran acceder a similares ritmos de crecimiento de su PIB per cápita, una vez alcanzado el estado estacionario. Es más, ese estado estacionario debiera ser el mismo para los países que compartan los parámetros relevantes, lo que debiera llevar aparejado un proceso de convergencia manifiesto en sus niveles de renta per cápita. Es claro que la primera de las consecuencias no se observa en la economía mundial. La segunda, por su parte, ha sido objeto de una intensa labor investigadora, con resultados que requieren precisiones ulteriores.

Las estimaciones no permiten validar la existencia de un proceso de convergencia absoluta (que comportaría una obligada reducción de las desigualdades internacionales). Como señala Barro (1991: 407), "la hipótesis de que los países pobres tienden a crecer más rápido que los países ricos parece inconsistente con la evidencia que emerge de los estudios cross-country, que indica que las tasas de crecimiento per cápita tienen una limitada correlación con el nivel del ingreso per cápita de partida". Parece confirmarse, sin embargo, la existencia de un proceso de convergencia condicional (que comporta la aproximación sólo entre países que comparten estado estacionario) $)^{2}$. De hecho, este último resultado ha sido presentada por Sala-iMartin, $(2002 ; 6)$ como "uno de las más sólidas y robustas regularidades empíricas encontradas en los datos". No obstante, ese proceso incluso es discutible que se produzca en todos los casos. De forma más precisa, la evidencia internacional no es unánime y parece sugerir que la eficacia del proceso de convergencia es dispar, de acuerdo con el nivel de desarrollo del grupo de países considerado. En concreto, para los niveles de renta más baja hay evidencia de divergencia (más que de convergencia), mientras que esta última se hace más visible en los casos de los países de renta media y alta. Lo que sugeriría que el proceso está lejos de ser lineal.

Retornando a las prescripciones originarias del modelo de Solow, sólo cabe interpretar que las acusadas diferencias que se observan en los niveles de desarrollo de los países son consecuencia bien de la diversa ubicación de los países en relación con su respectivo estado estacionario (distintas fases de su transición), bien de la existencia de estados estacionarios diferentes. En el primer caso, la hipótesis claramente sobre-estima las posibilidades dinámicas de los países más pobres, respecto a lo observado internacionalmente. Un resultado tanto más paradójico si se tiene en cuenta que la mayor libertad en los movimientos de capital debiera acelerar los procesos de convergencia, cosa que, sin embargo, no se observa. En el segundo caso, es manifiesto que la discrepancia en los valores de los parámetros básicos que condicionan el estado estacionario en el modelo de Solow está muy lejos de poder explicar las diferencias que se observan en los niveles de renta de los países. Lo que obliga,

\footnotetext{
${ }^{2}$ En el primer caso, la estimación es del tipo $y_{i, t, t+T}=\beta_{o}-\beta_{1} \ln Y_{i t}+\varepsilon_{i t}$; por su parte, en el caso de la convergencia condicional, la estimación es del tipo $y_{i, t, t+T}=\beta_{0}-\beta_{1} \ln Y_{i t}+\beta_{2} \ln Y_{i}^{*}+\varepsilon_{i t}$. Donde y es la tasa de crecimiento del PIB per cápita, Y es el nivel del PIB per cápita del país en cuestión e $\mathrm{Y}^{*}$ expresa la renta per cápita del estado estacionario. En el primer caso se considera que todos los países comparten el mismo estado estacionario (o, al menos, que tal estado no depende del nivel de renta).
} 
necesariamente, a considerar que existen entre los países diferentes capacidades de producción (o de absorción) del progreso tecnológico, sin que el modelo dé explicación de esta discrepancia.

Mankiw et al. (1992) intentan aminorar la discrepancia observada, considerando las diferencias en los niveles de acumulación de capital humano entre países. En este caso, los bajos niveles de desarrollo de los países más pobres derivarían de la presencia, simultánea, de bajas tasas de inversión, bajos logros educativos y bajos niveles tecnológicos. La capacidad explicativa del modelo mejora notablemente al añadir este factor. Aun así, para que los planteamientos del modelo sean creíbles, las diferencias salariales entre mano de obra cualificada (capital humano) y no cualificada y los rendimientos de la educación deberían ser mucho más altas de las que habitualmente se observan. Tal como Hall y Jones (1999) demuestran, las diferencias en los niveles de capital físico y de logros educativos sólo pueden explicar parcialmente las diferencias en los niveles de output por persona entre países. Por otra parte, utilizando parámetros razonables, Romer (1995) confirma que para que el modelo dé los resultados apetecidos habría que aceptar, por ejemplo, que la tasa de rendimiento de la educación en los países en desarrollo debía ser 100 veces mayor que la de Estados Unidos; y la ratio entre salarios de mano de obra cualificada y no cualificada debería ser en el primer caso cerca de 200 veces la de Estados Unidos. Ninguno de estos supuestos parece razonable. Así pues, aun cuando existen diferencias notables en los logros educativos, no parece que sean de la dimensión requerida para explicar la magnitud de las desigualdades entre los niveles de renta de los países a escala internacional. Por lo demás, los resultados del modelo son altamente sensibles a los modos de aproximar empíricamente la acumulación de capital humano (Klenow y Rodríguez-Clare, 1998 o Dinopoulos y Thompson, 1998).

No son mucho más sólidos los resultados de aquellos estudios que han tratado de comprobar la validez de la nueva teoría del crecimiento (Sala-i-Martin, 1997, o Durlauf y Quah, 1999). En primer lugar, los modelos que suponen rendimientos constantes en el capital (tipo AK) resultan poco acordes con los estudios sobre la convergencia económica, salvo que se considere que el proceso de convergencia se produce por vía de difusión tecnológica, más que a través de la creciente capitalización de la economía. En segundo lugar, aquellos modelos en los que se incorpora un factor de escala en la determinación del crecimiento del estado estacionario (como Romer, 1986 o Romer, 1990) parecen difíciles de interpretar, salvo que se refieran al conjunto de la economía mundial (y no tanto a países singulares). En tercer lugar, tanto en estos casos como en el de Lucas (1988), que admite linealidad del output en el capital humano, parece difícil compatibilizar la expansión que se ha producido en la dotación de capital tecnológico y humano a escala internacional con la inmutabilidad de la tasa de crecimiento en el tiempo. De hecho, como es bien sabido, la tasa de crecimiento de las economías de la OCDE durante las tres últimas décadas (donde ha sido mayor el esfuerzo educativo y tecnológico) es menor que la registrada en el cuarto de siglo precedente (en la llamada "edad de oro" del capitalismo, entre 1950 y 1973). 
Una de las implicaciones adicionales de los modelos de crecimiento endógeno es que la dinámica económica puede quedar condicionada por las políticas económicas aplicadas por el país en cuestión. No obstante, tampoco aquí las evidencias resultan concluyentes. Es bien conocido el trabajo que realizaron Levine y Renelt (1992) para tratar de comprobar el grado de robustez de las variables normalmente asociadas al crecimiento económico, cuando estas variables se incorporan en diversas especificaciones. Pues bien, de todas las consideradas en su amplio escrutinio, solamente la inversión supera la prueba establecida. Esto podría suponer un respaldo al modelo Harrod-Domar, pero como revelan King y Levine (1994), la relación parece operar en sentido contrario al previsto por la teoría: es el crecimiento el que impulsa la inversión, más que la inversión la que promueve el crecimiento.

Unos años más tarde, Sala-i-Martin (1997) repitió el ejercicio, pero estableciendo una prueba menos exigente para medir la robustez de las variables. En el conjunto de las 21 variables que superan su test se incluye un amplio grupo de factores estructurales (como la ubicación regional del país, su filiación religiosa o su latitud geográfica), del clima político (como derechos políticos y civiles, respeto a la ley o revoluciones) y algunas más directamente relacionadas con la actividad económica (como inversión, número de años que lleva el país abierto al comercio o 'grado de capitalismo' del país). De todas estas variables, sólo dos parecen conectar con lo que constituye la teoría de crecimiento, aun cuando su conexión está lejos de ser nítida: los años que el país lleva considerándose una economía abierta y el 'grado de capitalismo' propio de la economía. Como se ve, el balance de resultados no es excesivo, como el propio Sala-i-Martin (2002) reconoce. No es extraño, por tanto, que Kenny y Williams (2001: 1) concluyan que "si la evidencia muestra algo, es que muy diferentes combinaciones de políticas pueden ser apropiadas para diferentes países, en diferentes momentos".

La acumulación de estos resultados puede ser consecuencia, sin duda, de la baja calidad de la información empírica o de las limitaciones de los procedimientos estadísticos y econométricos utilizados. No obstante, parece que además de estos potenciales problemas existen otros que se relacionan con el propio modo de entender (y estudiar) el crecimiento. De especial relevancia es, al respecto:

i) en primer lugar, el afán por buscar causas únicas explicativas del crecimiento, aptas para toda etapa de desarrollo y para todo lugar;

ii) en segundo lugar, la pretensión de linealidad y causalidad de sentido único en la acción de los factores, excluyendo la existencia de umbrales, discontinuidades y relaciones circulares; y

iii) en tercer lugar, el propósito de definir una condición de equilibrio, única y estable.

Estos supuestos, que subyacen a toda la teoría el crecimiento, fueron sin embargo puestos en cuestión por la teoría del desarrollo, desde sus mismos orígenes, por considerarlos poco compatibles con la experiencia real de los países en desarrollo. 


\section{La teoría del desarrollo}

En sus inicios, al final de los cuarenta, la economía del desarrollo se intentó establecer como una disciplina diferenciada de la corriente teórica principal de la economía. En sus primeras caracterizaciones, los países en desarrollo aparecen como economías con abundancia de mano de obra y bajos salarios, pero también con limitada dotación de capital y bajos rendimientos de capital. En el caso de Nurkse (1953), esa baja dotación de capital está altamente relacionada con la reducida capacidad de ahorro de los países en desarrollo, debido a su bajo nivel de renta, pero también con el bajo rendimiento del capital (la "debilidad de los incentivos a la inversión", en términos de Nurkse). Es la combinación de estos dos hechos, bajo rendimiento de capital y de trabajo, lo que reclamaba una explicación específica para los países en desarrollo, que apareció asociada a la definición de la "trampa de la pobreza", acaso el elemento de diagnóstico más compartido entre los teóricos del desarrollo.

La justificación de la trampa de la pobreza descansa en la presencia de relaciones circulares (no meramente unidireccionales) entre las variables relevantes y la eficacia de importantes externalidades (pecuniarias y tecnológicas) y rendimientos crecientes en la actividad económica. La presencia de externalidades en la actividad económica será, de hecho, un elemento caracterizador de buena parte de las propuestas en este campo, como las debidas a Hirschman (1958), Myrdal (1957), RosesteinRodan (1943) o Lewis (1954). La combinación de importantes externalidades con la presencia de indivisibilidades en la inversión, conduce, a la vez, a la ruptura de la linealidad, dando lugar a la posible presencia de múltiples equilibrios a los que se enfrenta la trayectoria de una economía (Rosestein-Rodan, 1943, Leibenstein, 1954 o Nelson, 1956). Y, en fin, la combinación de esos factores hace que la senda previa seguida por una economía condicione su posible evolución futura, haciendo que la historia cuente. Como señaló Myrdal $(1957 ; 16)$ resumiendo esta visión, el problema del desarrollo está relacionado con "un complejo sistema de cambios que están interrelacionados, son circulares y de carácter acumulativo".

Haciendo un esfuerzo de síntesis, la especificidad de la explicación inicial del subdesarrollo descansó, muy centralmente, sobre tres factores: en primer lugar, la conspicua existencia de rendimientos crecientes y de externalidades (pecuniarias y tecnológicas) en la vida económica; en segundo lugar, la presencia de complementariedades e indivisibilidades en la actividad inversora; y, en tercer lugar, en la existencia de una oferta laboral plenamente elástica, dada la presencia de excedentes de mano de obra procedentes de un sector tradicional y superpoblado que convive con un minúsculo sector moderno orientado al mercado. La combinación de estos tres elementos conducía a la existencia de múltiples equilibrios y a las dificultades para transitar entre ellos. Como, con acierto, señala Ros (2000: 4): "una dosis moderada de rendimientos crecientes a escala combinada con la presencia de un excedente de mano de obra puede motivar una radical diferencia respecto al modelo neoclásico".

No obstante, sería excesivo presentar la teoría del desarrollo como un corpus teórico único e integrado: de hecho, más que hablar de "teoría del desarrollo" habría 
que hablar de "teorías del desarrollo". Pues, en efecto, más allá de algunos postulados comunes, lo cierto es que bajo aquel rótulo se acoge una amplia colección de interpretaciones no necesariamente coincidentes. En esto se distancia la teoría del crecimiento de la propia del desarrollo: mientras la primera descansa en un núcleo doctrinal generalmente compartido, aportado en su día por Solow, la segunda se compone de interpretaciones dispares y, en algunos casos, contradictorias. Sin duda, esta ausencia de un núcleo paradigmático -en términos de Lakatos- ha debilitado la capacidad de la teoría del desarrollo para imponerse como interpretación dominante de la dinámica económica. En esa colección de interpretaciones que nutren la teoría del desarrollo hay, sin embargo, un puñado de intuiciones inspiradoras, de notable capacidad de sugerencia, que han resistido el paso del tiempo. Es cierto que gran parte de esas intuiciones se presentaron de forma poco adecuada para derivar de ellas modelos precisos, susceptibles de contrastación, pero aún así tienen una capacidad heurística notable.

Si hubiera que rescatar las intuiciones más valiosas, tres serían las que concentrarían nuestra atención: la importancia del cambio estructural, el relevante papel de las externalidades y la existencia de múltiples equilibrios. Veamos brevemente estos aspectos.

a) La importancia del cambio estructural

La teoría del desarrollo considera inadecuada aquella aproximación al crecimiento que parte de considerar el producto como un flujo de bienes y servicios invariante en el tiempo. El cambio en el peso de los sectores económicos a medida que se avanza en los niveles de renta per cápita constituye una de las regularidades del proceso de crecimiento. Este proceso no es ni aleatorio ni irrelevante para la dinámica de crecimiento. Como demostraron Kuznets (1960) o Chenery y Syrquin (1975) los cambios en la oferta responden a pautas bien establecidas y relativamente comunes; al tiempo, ese cambio no sólo es consecuencia del crecimiento, sino también causa de posibles alteraciones en la dinámica económica (Baumol, 1967). Al contrario que los teóricos del crecimiento, los economistas del desarrollo tomaron muy en cuenta este proceso de cambio, formando parte central de las propuestas argumentadas por Rostow (1961) y Lewis (1954). En general, en todos los casos se insiste en el papel crucial que la industria tiene en la transición desde un estado de bajo crecimiento a una economía dinámica. Kaldor (1967: 7) se hará eco de esta visión: "elevadas tasas de crecimiento del producto están casi ineludiblemente asociadas con elevadas tasas de crecimiento del sector secundario, principalmente, de las manufacturas". Contemplar la relevancia del cambio estructural no sólo supone incorporar un elemento adicional en la explicación de la dinámica económica, sino también abre la posibilidad a considerar potenciales cambios en los factores promotores del crecimiento de acuerdo con la etapa de desarrollo en la que se encuentre el país en cuestión. 
b) La presencia de externalidades e indivisibilidades en la inversión

Una segunda aportación relevante de la teoría del desarrollo es el papel crucial que tienen los efectos inducidos e indirectos (externalidades y complementariedades) de las distintas actividades económicas. Es el papel de esas externalidades lo que justifica la propuesta de Myrdal (1957) acerca de la "causación acumulativa", el análisis que Hirschman (1958) hace acerca de los eslabonamientos interindustriales o la propuesta de Rosestein-Rodan (1943) acerca del 'big push'. Este último, además insiste en la presencia de indivisibilidades en la inversión, lo que obliga a considerar la existencia de umbrales en la interconexión eficiente entre las variables (Rosestein-Rodan, 1984). En todos estos casos, la inversión genera un efecto sobre actividades conexas, generando una dinámica inducida que es necesario considerar para entender el proceso de crecimiento. La presencia de estos efectos indirectos tiene una consecuencia relevante para la estrategia de desarrollo: el mercado puede no ser capaz de garantizar, por sí mismo, una solución óptima: un planificador puede conseguir, bajo determinadas condiciones, un resultado más eficiente. En especial, un marco institucional adecuado puede ayudar a resolver los problemas de coordinación que comporta la presencia de externalidades para la acción autónoma de los agentes económicos. Un planteamiento que contrasta con el marco doctrinal en el que preferentemente descansa la teoría del crecimiento. Es cierto que ésta última ha logrado también integrar en alguno de sus modelos el efecto de las externalidades, especialmente como vía para incorporar un progreso técnico endógeno compatible con las condiciones de competencia perfecta. No obstante, incluso en ese caso se respeta la linealidad de las relaciones básicas, eludiendo la presencia de discontinuidades (o umbrales) en el comportamiento económico.

c) La existencia de múltiples equilibrios

Por último, derivado de los dos rasgos anteriores, una parte de la teoría del desarrollo se construyó aceptando la potencial existencia de múltiples equilibrios. En ese caso, el proceso de desarrollo se asemeja a un problema de optimización con múltiples restricciones, que en muchos casos no operan simultáneamente, sino que se hacen evidentes una vez que una restricción previa es eliminada. En concreto, la teoría del desarrollo nació preocupada por la existencia de trampas de equilibrio de bajo nivel (low-level equilibrium traps): es decir, equilibrios localmente estables pero compatibles con la existencia de otros equilibrios a los que es posible acceder siempre que se venzan las resistencia de la estabilidad local (Leibenstein, 1954). Los trabajos clásicos de Nurkse (1957) sobre las trampas de pobreza y el de RosenteinRodan (1943) sobre el 'big push' constituyen ejemplos claros de situaciones donde una economía se puede encontrar en un equilibrio estable negativo. Desde esta perspectiva, la teoría del desarrollo más que en definir las condi- 
ciones universales de un estado estacionario (steady state), parece interesada en explicar las causas de los desequilibrios, la potencial existencia de más de un estado estacionario y la compleja transición de uno a otro. RosesteinRodan (1984: 207) lo señaló claramente: "un análisis del desequilibrio del proceso de crecimiento es lo que es esencial para entender los problemas del desarrollo económico".

Los rasgos del proceso de desarrollo que más arriba se han señalado justifican que se consideren las instituciones como un factor relevante en la promoción de la dinámica económica (Alonso y Garcimartín, 2008). Los problemas de coordinación en la acción inversora, de aprovechamiento de externalidades y de superación de equilibrios de bajo nivel ponen en evidencia las limitaciones de confiar en la mera acción individualizada de los agentes a través del mercado; $y$, al contrario, subrayan la potencial necesidad de una cierta forma de acción colectiva, no incompatible con el mercado, que se erige a través de las instituciones. No es extraño, entonces, que la literatura sobre el desarrollo haya prestado una especial atención al papel que las instituciones tienen en la estrategia de desarrollo. Lo señaló ya hace más de cuatro décadas Myrdal (Myrdal, 1974: 21):" las actitudes e instituciones son más importantes que los niveles de ingreso por sí solo". Los estudios empíricos han otorgado respaldo a esta observación de Myrdal.

La categoría analítica más difundida que emana de estos planteamientos es la de "trampa de la pobreza". Pese a que su origen está en los trabajos pioneros del desarrollo de la década de los años cincuenta, hubo que esperar hasta la segunda mitad de los años ochenta para que esa categoría y la existencia derivada de múltiples equilibrios adquiriesen una expresión formal consistente (Murphy et al., 1989, Azariadis, 2006 o Bowles et al., 2006) La contribución de estos trabajos más recientes no consiste tanto en la novedad de su contenido como en la sólida e ingeniosa fundamentación que ofrecen de la propuesta y en su extensión a una gran variedad de situaciones. Cabría sistematizar esos modelos en torno a tres grandes agregados (Bowles et al., 2006):

i) la presencia de externalidades y de rendimientos crecientes localizados (thresholds models of poverty);

ii) la presencia de un marco institucional inadecuado, con problemas para la acción colectiva (institutions as poverty trap); y

iii) y la existencia de efectos de vecindad que condicionan el comportamiento agregado (neighborhood effets as poverty traps).

La literatura especializada confirma la pluralidad de causas que pueden estar detrás de una trampa de pobreza: una situación en la que confluyen una circularidad de factores obstaculizadores del progreso. Un detalle más preciso de aquellos factores que están en el origen de trampas de la pobreza serían, sin ánimo de exhaustividad, los siguientes (Azariadis, 2006): 
i) Impaciencia en el comportamiento ya sea de los gobiernos, debido a los niveles de corrupción, ya de las familias con limitada esperanza de vida, debido a los bajos niveles de salud, o ya de agentes con niveles elevados de inseguridad respecto al valor fututo de su riqueza acumulada, por la inestabilidad económica o social (Azariadis, 2006). En todos estos casos la situación de la economía conduce a un incremento del consumo a costa de la capacidad inversora y, por consiguiente, del crecimiento.

ii) Distorsiones en la actividad financiera debido ya a altos costes de intermediación ya a la limitada diversificación de actividades, lo que dificulta que se genere una oferta de aseguramiento adecuada (Azariadis, 2006 o Saint-Paul, 1992). Las imperfecciones en los mercados de capital y de aseguramiento, limitan también la capacidad inversora y alientan un uso ineficiente del ahorro.

iii) Elevado crecimiento demográfico en un contexto de baja renta familiar y con comportamiento endógeno de la fertilidad (Nelson, 1957 o Becker, Murphy y Tamura, 1990). La expansión demográfica en este contexto dificulta el logro de progresos en términos materiales.

iv) Baja elasticidad de sustitución técnica entre capital y trabajo en los niveles de bajo desarrollo (De la Croix y Michel, 2002), lo que dificulta la flexibilidad de la economía y la adopción de tecnologías más productivas.

v) Fuertes rendimientos crecientes locales (o thresholds) en la producción de bienes, pero especialmente en la investigación e innovación o en la formación de capital humano (Diamon, 1982 y Azariadis y Drazen 1990). En la medida en que existan complementariedades entre capital físico y humano, esto conducirá, además, a un menor rendimiento del capital invertido, en un contexto de bajos niveles de formación.

vi) Rendimientos de escala dinámicos (learning by doing externalities), como se argumenta en el caso de la industria naciente o en ámbitos relacionados con el conocimiento y el capital humano (Stokey 1988, Matsuyama, 2002). Este factor limitaría las posibilidades de desarrollo en ámbitos de industriales intensivos en conocimiento.

vii) Problemas asociados a la limitada especialización productiva y al impulso dinámico que generan las demandas recíprocas, tal como en su momento señaló Rosestein-Rodan (1943) y desarrollan Murphy et al. (1989).

viii) Contagio de normas sociales que condicionan el comportamiento de cada agente (Todaro, 1996), como es el caso de la resistencia a otorgar confianza a las instituciones (cuando el sentimiento de desconfianza es generalizado) o de cambios en las conductas corruptas (cuando se trata de una práctica asentada, Bhardan et al., 2000).

Muchos de los factores señalados como potenciales causas de una "trampa de la pobreza" se presentan de forma simultánea en los países pobres, lo que hace más difícil poner en marcha estrategias para superarlos. Al fin, a este tipo de países les 
caracteriza una deficiente estructura institucional, la carencia o estrechez de los mercados financieros domésticos, los efectos de la inseguridad sobre el comportamiento de los agentes, la muy fragmentada especialización productiva o la corrupción de la Administración, por señalar alguno de los más relevantes. Esta diversidad de problemas hace difícil tanto el diagnóstico como la terapia del desarrollo propia de estos países.

Por lo demás, asumir la presencia de este tipo de fenómenos tiene serias consecuencias en el diseño de las políticas y para los ejercicios empíricos derivados. Por lo que se refiere al primer aspecto, la presencia de equilibrios múltiples:

i) hace, en primer lugar, que las condiciones de las que se parte cobren una gran importancia ${ }^{3}$;

ii) en segundo lugar, los efectos de las políticas económicas pueden verse amplificados con el transcurso del tiempo, al situar a las economías en una senda hacia un equilibrio estable (ya sea positivo o negativo) ${ }^{4}$; y

iii) en tercer lugar, que los desplazamientos dosificados de un equilibrio estable puedan no tener efectos sostenibles en el tiempo, ya que la tendencia sea volver al punto de equilibrio inicial (ya sea este positivo o negativo).

En suma, el hecho de que cada economía transite hacia un equilibrio diferente implica que no existe una política económica única y universal: no se trata de que la "dosis" aplicable pueda diferir de unos países a otros, sino de que la propia "receta" puede ser distinta.

Por lo que se refiere a las dificultades del trabajo empírico, el principal problema deriva del tipo de relaciones -no lineales y circulares- que se presupone entre las variables. Las técnicas econométricas más al uso están construidas sobre la base de captar relaciones lineales, donde la causalidad es de única dirección. Superar estos problemas supone una dificultad adicional para traducir los modelos a estimaciones robustas $^{5}$.

\section{Consideraciones finales}

La teoría del desarrollo parte de considerar como limitada aquella concepción que asimila el crecimiento con la suma de acumulación de factores y cambio técnico. En su concepción, la dinámica de desarrollo no sólo comporta acumulación, sino también reasignación productiva, que es la fuente del cambio técnico, aprovecha-

\footnotetext{
${ }^{3}$ Por ejemplo, dos países con valores semejantes en una cierta variable pueden experimentar una evolución divergente por el simple hecho de que ambos se encontraban inicialmente a ambos lados de un equilibrio inestable.

${ }^{4}$ Como corolario y teniendo en cuenta el primer punto, diferencias en las políticas económicas consideradas como poco relevantes pueden ser determinantes de sendas de desarrollo diferentes.

${ }^{5}$ Emplear especificaciones con productos de variables o con potencias no resuelve este problema.
} 
miento de las externalidades, mejoras en la coordinación para superar las indivisibilidades y las complementariedades de la inversión y posible existencia de múltiples equilibrios con sendas dispares de evolución que influyen en el resultado final (path dependence).

Pues bien, algunas de las intuiciones que alimentan esta visión podrían ser enormemente inspiradoras para superar alguna de las limitaciones de la teoría del crecimiento. Así, por ejemplo, Durlauf y Johnson (1995) y Dinopoulos y Thompson (1999) argumentan la necesidad de considerar la presencia de capacidades tecnológicas y preferencias diversas de acuerdo con el nivel de desarrollo de los países, sugiriendo la conveniencia de discriminar las muestras de países en las estimaciones de funciones de crecimiento. Y Greiner et al. (2005) observan que la capacidad explicativa de los diversos modelos de crecimiento varían de acuerdo con el nivel de desarrollo de los países: "en los primeros estadios, aprender de otros, externalidades y economías de escala son las principales fuentes del crecimiento económico. En un estadio posterior, la educación y la creación de capital humano son importantes, hasta el punto de que el crecimiento parece ser proporcional a los esfuerzos dedicados a la educación" (Greiner et al., 2005: 2). Estos mismos autores argumentan la posible presencia de no linealidades en el comportamiento de las variables para explicar los efectos de escala contemplados en alguno de los modelos de crecimiento endógeno; y sugieren la presencia de indivisibilidades en la función de producción, que se expresa como convexa-cóncava para expresar rendimientos crecientes en una primera etapa y rendimientos decrecientes más adelante (una sugerencia propuesta también por Durlauf y Quah, 1999). Son muestras de cómo algunas instituciones de la teoría del desarrollo se han integrado ya en la modelización del crecimiento. Se trata de un camino en el que se debiera proseguir.

\section{Bibliografía}

[1] AGHION, P. y P. HOWITT (1992): "A model of economic growth through creative destruction", Econometrica, 60, pp. 323-35.

[2] AGHION, P. y P. HOWITT (1998): Endogenous growth theory, Cambridge, MIT Press.

[3] ALONSO, J.A. y C. GARCIMARTÍN (1988): Acción colectiva y desarrollo. El papel de las instituciones, Madrid, Editorial Complutense.

[4] ARROW, K.J. (1962): "The economic implications of learning by doing", Review of Economic Studies, 29, pp. 155-173.

[5] AZARIADIS, C. (2006): "The theory of Poverty Traps. What have we Learned?" en Bowles, Durlanf y Hoff (2006), Poverty Traps, Princeton, Princeton University.

[6] AZARIADIS, C. y A. DRAZEN (1990): “Threshold Externalities in Economic Development”, Quarterly Journal of Economics, 105: pp. 521-26.

[7] BARRO, R.J.: "Economic growth in a cross section of countries", Quarterly Journal of Economics 106, 1991, pp. 407-443.

[8] BAUMOL, J. (1967): "Macroeconomics of unbalanced growth: The anatomy of the urban crisis", American Economic Review, 57, 2, pp. 415-426 
[9] BECKER, G.S., K. M. MURPHY y R. TAMURA (1990): "Human capital, Fertility and Economic Growth", Journal of Political Economy 98 (5), pp. 512-537.

[10] BOWLES, S., S.N. DURLANF y K. HOFF (2006): Poverty Traps. Princeton. Princeton University Press.

[11] CASS, D. (1965): "Optimun growth in an aggregative model of capital accumulation", en The Econometric Approach to Development Planning, Amsterdam, NorthHolland.

[12] CHENERY, H.B. y M. STROUT (1966): "Foreign assistance and economic development", American Economic Review, 56, pp. 679-733

[13] CHENERY, H.B. y M. SYRQUIN (1975): Patterns of Development, 1950-1970, London, Oxford University Press

[14] DE LA CROIX, D. y P. MICHEL (2002): A Theory of Economic Growth, Cambridge, Cambridge University Press.

[15] DIAMOND, P. (1982): "Aggregate demand management in search equilibrium", Journal of Political Economy, 90, pp. 881-894.

[16] DINOPOULOS, E. y P. THOMSON (1998): "Schumpeterian growth without scale effects", Journal of Economic Growth, 3, pp. 313-335.

[17] DOMAR, E.D. (1946: "Capital expansion, rate of growth, and employment”, Econometrica, 14, pp. 137-147.

[18] DURLAUF, S. y D. QUAH (1999): “The new empirics of economic growth”, en J. Taylor y M. Woodford (editores): Handbook of Macroeconomics, vol. I, Amsterdam, Elsevier Science.

[19] DURLAUF, S.N. y P.A. JHONSON (1995): "Multiple regimes and cross-country growth behaviour ", Journal of Applied Econometrics, vol. 10, pp. 365-384.

[20] GREINER, A., W. SEMMIER y G. GONG (2005): The forces of economic growth. A time series perspective, Princeton, Princeton University Press.

[21] GROSSMAN, G.M. y E. HELPMAN (1991): Innovation and growth in the global economy, MIT Press, Cambridge, MA.

[22] HALL, R.E. y CH.I. JONES (1999): "Why do some countries produce so much more output per worker than others?" Quarterly Journal of Economics, vol 114 (February), pp. 83-116.

[23] HARROD, R. (1939): An essay in dynamic theory", Economic Journal, 49, pp. 14-33.

[24] HARROD, R. (1948): Towards a Dynamic Economics, London, Macmillan.

[25] HIRSCHMAN, A.O. (1958): The strategy of economic development, New Haven, Yale University Press.

[26] KALDOR, N. (1957): “A model of economic growth”, Economic Journal, 67, pp. 591624.

[27] KALDOR, N. (1967): Strategic Factors in Economic Development, Ithaca, Cornell University.

[28] KALDOR, N. y J.A. MIRRLEES (1962): “A new model of economic growth", The Review of Economics and Statistics, 29, pp. 174-192.

[29] KENNY, CH. y D. WILLIAMS (2001): "What do we Know about economic growth? Or, why don't we know very much?", World Development, 29, 1.

[30] KING, R. y R. LEVINE (1994): "Capital fundamentalism, economic development, and economic growth", Policy Research Working Paper 1462, World Bank. 
[31] KLENOW, P.J. y A. RODRÍGUEZ-CLARE (1998): “The neoclassical revival in growth economics: has it gone too far?", en B. Bernanke y J.J. Rotemberg (eds), NBER Macroeconomics Annual 1997, Cambridge Mass., MIT Press.

[32] KOOPMANS, T.C. (1965): "On the concept of optimal economic growth", en The Econometric Approach to Development Planning, Amsterdam, North-Holland.

[33] KUZNETS, S. (1960): "Quantitative aspects of the economic growth of nations, V: capital formation proportions: International comparisons for recent years", Economic Development and Cultural Change, vol 6.

[34] LAUDAN, L. (1977): Progress and its problems, Berkeley, University of California Press.

[35] LEIBENSTEIN, H. (1954): Economic Backwardness and Economic Growth, New York, John Wiley and Sons.

[36] LEVINE, R. y D. RENELT (1992): "A sensitivity analisys of cross-country growth regressions", American Economic Review, 82 (4), pp. 942-963.

[37] LEWIS, A. (1954): "Economic development with unlimited supplies of labor", Manchester School of Economics and Social Studies.

[38] LUCAS, R.E. (1988): "On the Mechanics of Economic Development," Journal of Monetary Economics 22, pp. 3-42.

[39] MANKIW, N.E., P. ROMER y D.N. WEIL (1992): "A contribution to the empirics of economic growth", Quarterly Journal of Economics, 107, pp. 407-437.

[40] MATSUYAMA, K. (2002): "The rise of mass consumption societies", Journal of Political Economy, 110, October, pp. 1035-1070.

[41] MILIKAN, M. y W.W. ROSTOW (1957): A Proposal: Key to an Effective Foreign Policy, New York, Harper and Brothers

[42] MURPHY, K.M., A. SHLEIFER y R. VISHNY (1989): “Income distribution, market size and industrialization", Quarterly Journal of Economics, 4, pp. 537-564,

[43] MYRDAL, G. (1957): Economic theory and underdeveloped regions, London, Duckworth.

[44] MYRDAL, G. (1974): La pobreza de las naciones, Ariel, Barcelona.

[45] NELSON, R. (1956): "A theory of the low level equilibrium trap", American Economic Review, 64 (5), May, pp. 894-908.

[46] NURKSE, R. (1957): Problems of Capital Formation in Underdeveloped Countries, New York, Oxford University Press.

[47] REBELO, S. (1991): "Long-run policy analysis and long-run growth", Journal of Political Economy, 99, pp. 500-521.

[48] RODRICK, D. (2004): “Getting Institutions Right”, en http.//ksghome.harvard.edu/ drodrik/.

[49] ROMER, P. (1986): “Increasing returns and long-run growth”, Journal of Political Economy, 94, pp. 1002-1037.

[50] ROMER, P. (1995): “Comment on Mankiw". Brookings Papers on Economic Activity, 1: 313-20.

[51] ROMER, P.M. (1990): "Endogenous technological change", Journal of Political Economy, vol. 98 m, pp. 71-102.

[52] ROS, J. (2000): Development Theory and the Economics of Growth, Ann Arbor, The University of Michigan Press. 
[53] ROSESTEIN-RODAN, P.N. (1943): "Problems of industrialization of Eastern and South-Eastern Europe", Economic Journal, 53, pp. 202-211.

[54] ROSESTEIN-RODAN, P.N. (1961): "International aid for underdeveloped countries", Review for Economic and Statistics, 43, pp. 107-138.

[55] ROSESTEIN-RODAN, P.N. (1984): "Natura facit saltum: Analysis of disequilibrium growth process", en G. Meier y D. Seers (eds), Pioneers in Development, New York, Oxford University Press.

[56] ROSTOW, W.W. (1961): The Stages of Economic Growth, Cambridge, Cambridge University Press.

[57] SAINT-PAUL, G. (1992): “Technology choice, financial markets and economic development”, European Economic Review, 36 (4), May, pp. 763-781.

[58] SALA-I-MARTÍN, X. (1992): "A positive theory of Social Security", Journal of Economic Growth 1, pp. 277-304.

[59] SALA-I-MARTIN, X. (1997): "I just run two million regressions", American Economic Review, 87, 2, pp. 178-183.

[60] SALA-I-MARTÍN, X. (2002): "15 years of new growth economics: What have we learnt?", Columbia University, Department of Economics, Discussion Paper Series 0102-47.

[61] SCHUMPETER, J.A. (1942): The theory of economic development, Cambridge Mass, Harvard University Press.

[62] SCOTT, M.F. (1989): A new view of economic growth, Oxford, Oxford University Press.

[63] SOLOW, R. (1956): A Contribution to the Theory of Economic Growth, Quarterly Journal of Economics, Vol. 70, No. 1, pp. 65-94.

[64] SOLOW, R. (1957): "Technical change and the aggregate production function", Review of Economics and Statistics, 39, pp. 312-320.

[65] SOLOW, R. (1994): "Perspectives on growth theory", Journal of Economic Perspectives, 8, pp. 45-54.

[66] STOKEY, N. (1988): "Learning-by-doing and the introduction of new goods", Journal of Political Economy, 96, August, pp. 701-717.

[67] SWAN, T.W. (1956): "Economic growth and capital accumulation", Economic Record, 32, pp. 334-361.

[68] TEMPLE, J. (1999): “The New Growth evidence”, Journal of Economic Literature, vol. 37, pp. 112-156.

[69] UZAWA, H. (1965): “Optimal technical change in an aggregative model of economic growth", International Economic Review, 6, pp. 18-31.

[70] YOUNG, A. (1928): "Increasing returns and economic progress", Economic Journal, 38, pp. 527-542. 